\title{
Highly nonlinear chalcogenide suspended-core fibers for applications in the mid-infrared
}

\author{
Enrico Coscelli ${ }^{a}$, Federica Poli ${ }^{a}$, Jianfeng Li $^{b, c}$, Annamaria Cucinotta ${ }^{a}$ and Stefano Selleri ${ }^{a}$ \\ ${ }^{a}$ Information Engineering Department, University of Parma, I-43124 Parma, Italy; \\ ${ }^{b}$ State Key Laboratory of Electronic Thin Films and Integrated Devices, School of \\ Optoelectronic Information, University of Electronic Science and Technology of China \\ (UESTC), Chengdu 610054, China; \\ ${ }^{c}$ Institute of Photonic and Technology (AIPT), Aston University, Birmingham, United \\ Kingdom
}

\begin{abstract}
Due to their unique dispersion and nonlinear properties, chalcogenide suspended-core fibers, characterized by a few micrometer-sized core suspended between large air-holes by few small glass struts, are excellent candidates for mid-infrared applications. In the present study the influence of the main cross-section characteristics of the chalcogenide suspended-core fibers on the dispersion curve and on the position of the zero-dispersion wavelength has been thoroughly analyzed with a full-vector modal solver based on the finite element. In particular, the design of suspended-core fibers made of both $\mathrm{As}_{2} \mathrm{~S}_{3}$ and $\mathrm{As}_{2} \mathrm{Se}_{3}$ has been optimized to obtain dispersion properties suitable for the supercontinuum generation in the mid-infrared.
\end{abstract}

Keywords: Microstructured fibers, Dispersion engineering, Supercontinuum generation, Nonlinear fibers, Chalcogenide optical fibers, Mid-infrared sources

\section{INTRODUCTION}

The Suspended-Core Fiber (SCF) is one of the most peculiar types of photonic crystal fibers, which has already found several applications, spanning from biological sample recognition, ${ }^{1,2}$ explosive detection, ${ }^{3}$ strain and temperature measurements, ${ }^{4}$ nonlinear frequency conversion ${ }^{5-7}$ and SuperContinuum (SC) generation. ${ }^{8}, 9$ All these applications exploit the unique properties of SCFs, which stem from the geometrical structure of the fiber crosssection. SCFs are characterized by a small glass core suspended between large air-holes by few, typically 3 to 6 , submicrometer-scale bridges. The core shape, large air-filling fraction and the huge numerical aperture provide very high light intensity in the core, which yields to strong nonlinear interaction, and cause a significant fraction of the power to be carried in the evanescent tails of the guided-mode field, allowing its exploitation for interaction with gases or liquids which can be infiltrated into the cladding air-holes. Furthermore, the strong dependence of the light confinement characteristics on both wavelength and core size have a significant impact on the dispersion profile, which can be significantly altered with respect to the one of the host material.

Among non-silica glasses, chalcogen element-based ones have recently attracted great attention because of their potential applications in telecommunications, such as signal regeneration, ${ }^{10}$ wavelength conversion in the Infrared (IR $)^{11,12}$ and mid-IR SC generation. ${ }^{13-15}$ Chalcogenide glasses are based on a mixture of chalcogen elements, that is sulphur, selenium, or tellurium, and other elements, such as arsenic, germanium, antimony, or gallium. Compared to silica glasses, they offer extraordinary nonlinear characteristics, i.e. their nonlinear refractive index can be 800 times higher than that of silica fiber, ${ }^{10}$ low two-photon absorption, ${ }^{16}$ and excellent transmission window that extends far into the IR spectral region. Chalcogenide glasses have also long material Zero Dispersion Wavelength (ZDW) compared to silica, which hinders their application in mid-IR nonlinear optics. Indeed, their large normal group velocity dispersion at telecom wavelengths is responsible of a quick degradation of short pulses, ${ }^{17}$ while the long ZDW does not allow anomalous dispersion pumping with conventional sources for broad

Further author information: (Send correspondence to Enrico Coscelli)

Enrico Coscelli: E-mail: enrico.coscelli@unipr.it

Micro-structured and Specialty Optical Fibres IV, edited by Kyriacos Kalli,

Jiri Kanka, Alexis Mendez, Proc. of SPIE Vol. 9507, 950707 · (c) 2015

SPIE · CCC code: $0277-786 X / 15 / \$ 18 \cdot$ doi: $10.1117 / 12.2178751$

Proc. of SPIE Vol. 9507 950707-1 


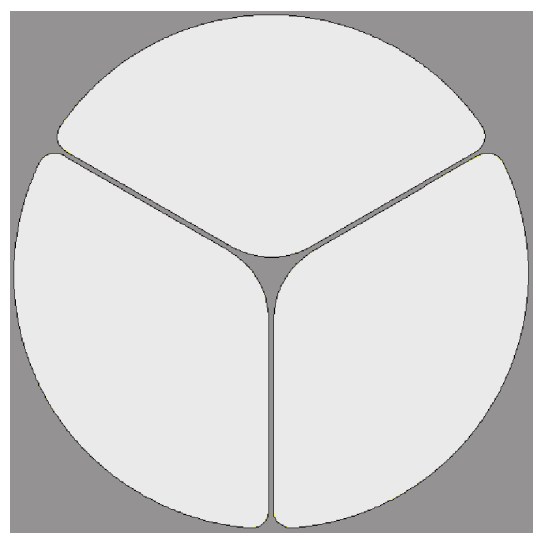

(a)

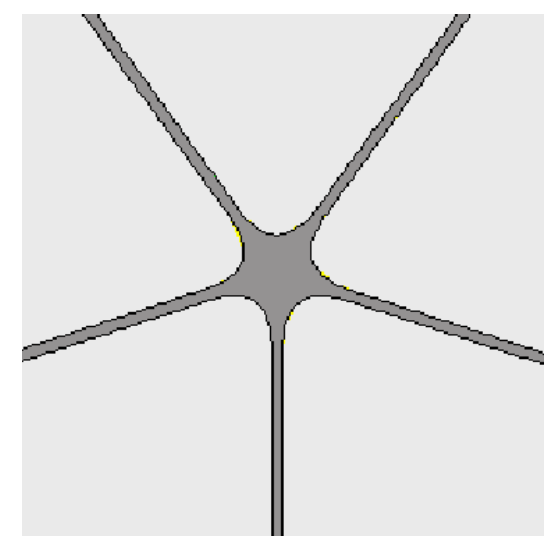

(b)

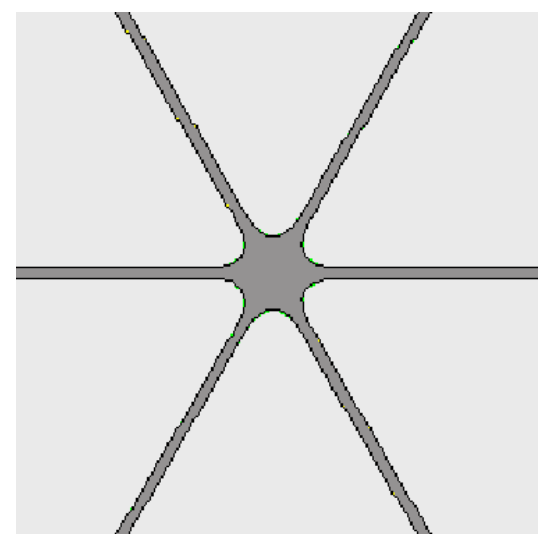

(c)

Figure 1. (a) Cross-section of the considered chalcogenide SCF with 3 bridges. Detail of the core of the SCF with (b) 5 bridges and (c) 6 bridges.

band SC generation.

Effective tailoring of the dispersion properties of chalcogenide fibers to the need of specific applications is therefore mandatory to be able to develop mid-IR optical devices. Thanks to the aforementioned properties, SCFs appear to be a valuable solution to this task. In this paper the dispersion properties of chalcogenide SCFs based on $\mathrm{As}_{2} \mathrm{~S}_{3}$ bulk glass have been thoroughly analyzed by taking into account the effects of all the main design parameters, such as core radius and strut number and thickness. Moreover, SCFs made of both $\mathrm{As}_{2} \mathrm{~S}_{3}$ and $\mathrm{As}_{2} \mathrm{Se}_{3}$ have been designed with ZDW around $2000 \mathrm{~nm}$ and $2800 \mathrm{~nm}$ in order to be exploited for the SC generation in the mid-IR. To this aim, a custom full-vector modal solver based on the finite-element method has been employed to calculate SCF guiding, dispersion and nonlinear properties. ${ }^{18}$

\section{SCF NUMERICAL MODELING}

Fig. 1(a) shows the cross-section of the more widespread SCF, that is the one with 3 air-holes in the cladding, separated by the same number of thin glass bridges, which create the core at their intersection. This structure is considered the starting point for the present study, since its main geometric characteristics have been changed in the numerical analysis, in order to evaluate their influence on the guiding properties and, consequently, on the dispersion parameter curve. The core radius $r_{c}$, which is the radius of the largest circumference inscribed into the core, the bridge thickness $t$ and their number have been modified in the SCFs. In particular, fibers with 5 and 6 glass bridges in the cross-section, shown in detail in Fig. 1(b) and (c), respectively, have been taken into account. In order to design technologically-feasible chalcogenide fibers or, at least, structures that can be obtained by tapering over lengths of several centimeters, ${ }^{8,19,20}$ values of $r_{c}$ in the range of $0.5 \mu \mathrm{m}-1.5 \mu \mathrm{m}$ and of $t$ between $0.15 \mu \mathrm{m}$ and $0.45 \mu \mathrm{m}$ have been considered for the simulations. The length of the struts has been fixed to about $20 \mu \mathrm{m}$, in order to prevent leakage of the fundamental mode into the cladding. Finally, two chalcogenide glasses, that is $\mathrm{As}_{2} \mathrm{~S}_{3}$ and $\mathrm{As}_{2} \mathrm{Se}_{3}$, providing the same good properties in terms of infrared transparency, nonlinearity and drawing capability ${ }^{19}$ but different contribution in terms of material dispersion, have been chosen for the numerical analysis. Notice that the refractive index of the chalcogenide glasses has been calculated according to the Sellmeier equation:

$$
n^{2}(\lambda)=1+\sum_{i} \frac{A_{i} \lambda^{2}}{\lambda^{2}-\lambda_{i}^{2}}
$$

Different values of the coefficients $A_{i}$ and $\lambda_{i}$ have been used for $\mathrm{As}_{2} \mathrm{~S}_{3}{ }^{21}$ and $\mathrm{As}_{2} \mathrm{Se}_{3} .{ }^{22}$

A full-vector modal solver based on the finite-element method ${ }^{18}$ has been applied to calculate the SCF fundamental mode in the wavelength range between $1000 \mathrm{~nm}$ and $3500 \mathrm{~nm}$. In order to investigate the nonlinear properties of the SCFs designed for the mid-IR SC generation, the nonlinear coefficient $\gamma_{N L}$ has been evaluated 

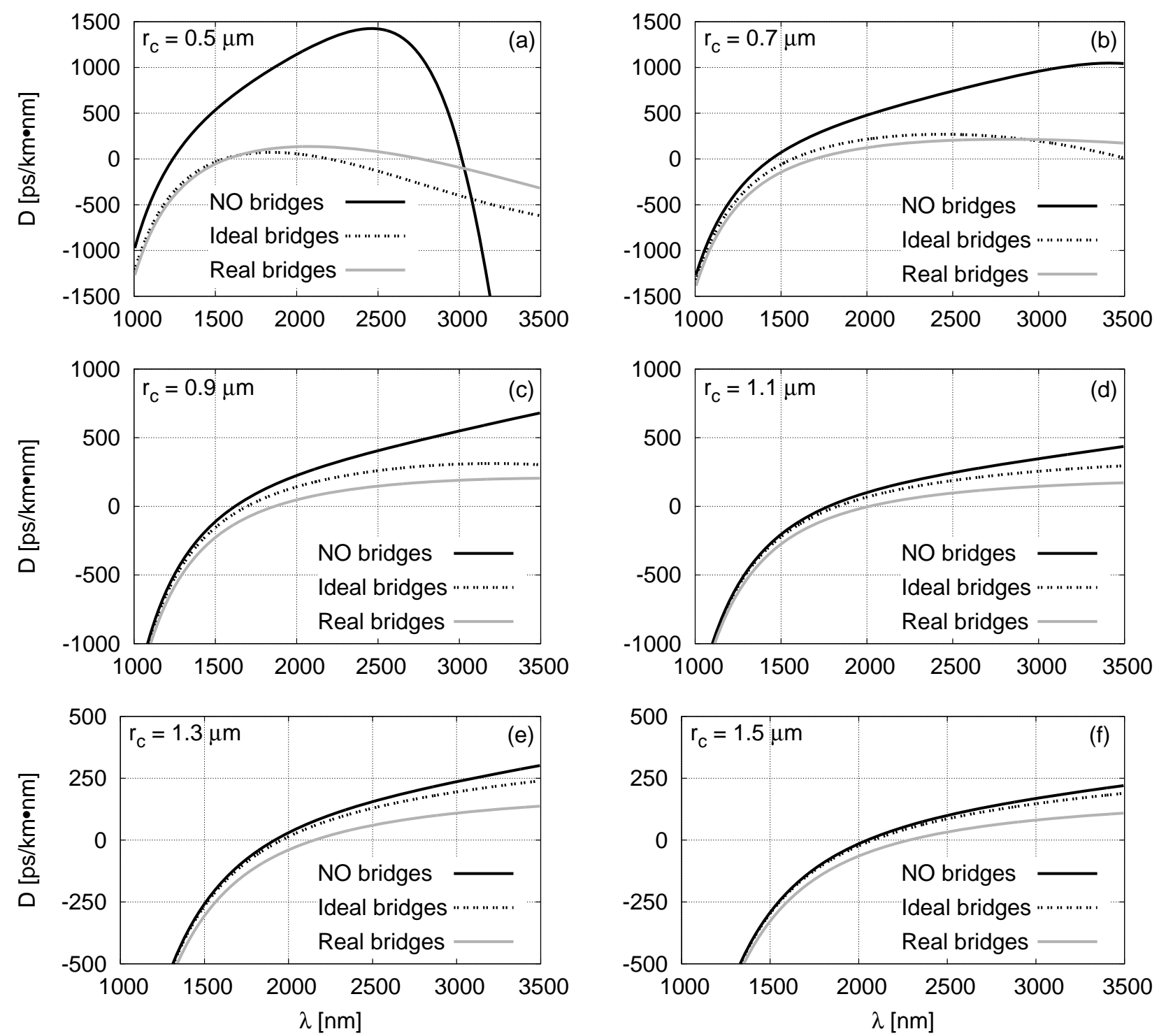

Figure 2. Dispersion parameter versus wavelength of the SCFs without bridges, with 3 ideal-shaped bridges and 3 realshaped bridges of thickness $t=0.45 \mu \mathrm{m}$, and core radius of (a) $0.5 \mu \mathrm{m}$, (b) $0.7 \mu \mathrm{m}$, (c) $0.9 \mu \mathrm{m}$, (d) $1.1 \mu \mathrm{m},(\mathrm{e}) 1.3 \mu \mathrm{m}$ and (f) $1.5 \mu \mathrm{m}$.

according to this formulation:

$$
\gamma_{N L}=\frac{2 \pi}{\lambda} \frac{n_{2, N L}}{A_{e f f, N L}}
$$

with

$$
A_{e f f, N L}=\frac{\left|\int_{S} \bar{E} \times \bar{H}^{*} \cdot \hat{z} d A\right|^{2}}{\int_{S}\left|\bar{E} \times \bar{H}^{*}\right|^{2} \cdot \hat{z} d A}
$$

being $S$ the glass domain in the SCF cross-section, ${ }^{23}$ and $n_{2, N L}$ the nonlinear refractive index, equal to $4.2 \cdot 10^{-18}$ $\mathrm{m}^{2} / \mathrm{W}$ and $1.5 \cdot 10^{-17} \mathrm{~m}^{2} / \mathrm{W}$ for $\mathrm{As}_{2} \mathrm{~S}_{3}{ }^{24}$ and $\mathrm{As}_{2} \mathrm{Se}_{3}$, respectively. Notice that the equation neglects the contribution to overall nonlinearity of air, which is 5 orders of magnitudes lower than the of the chalcogenide glasses. 

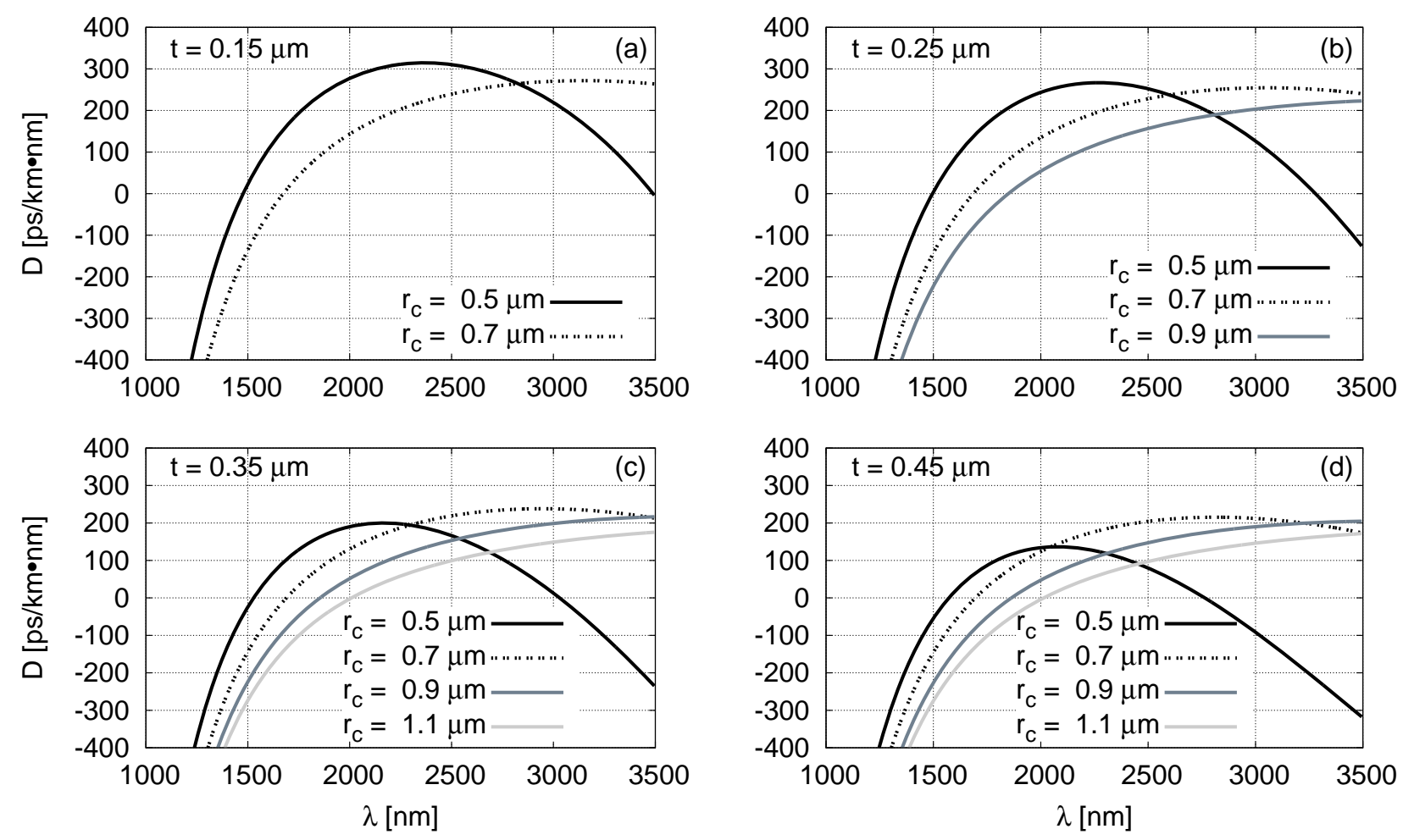

Figure 3. Dispersion parameter versus wavelength of the 3-bridge SCFs with strut thickness $t$ of (a) $0.15 \mu \mathrm{m}$, (b) $0.25 \mu \mathrm{m}$, (c) $0.35 \mu \mathrm{m}$, and (d) $0.45 \mu \mathrm{m}$, and different values of the core radius $r_{c}$.

\section{ENGINEERING OF SCF DISPERSION PROPERTIES}

The study of the influence of the bridge presence on the SCF dispersion properties represents the first step of this numerical analysis. To this aim, the dispersion parameter curve of $\mathrm{As}_{2} \mathrm{~S}_{3} \mathrm{SCFs}$ with 3 bridges $45 \mu \mathrm{m}$ wide has been calculated for different $r_{c}$ values, and compared in Fig. 2(a)-(f) with the one of a nanowire with the same radius, corresponding to the microstructured fiber core or, similarly, to the SCF without the struts. Moreover, the geometric characteristics of the region where the bridges connect to the core have been changed in order to analyze the role of the core shape on the SCF dispersion properties. In particular, SCFs with "real" bridges, that is linked to the core by parabolic-shaped connectors like in the fabricated fibers, as shown in Fig. 1(a), have been studied together with SCFs with "ideal" bridges, that is connected to the core in an abrupt way, without any transition region. Notice that with such a strut geometry, a smaller glass area is obtained for the core, for a fixed $r_{c}$ value, since all the interconnecting regions are missing.

Simulation results reported in Fig. 2 demonstrate that the bridge presence strong alters the SCF dispersion curve, especially when the core area is small. For example, as shown in Fig. 2(a), when $r_{c}=0.5 \mu \mathrm{m}$, the $D$ parameter curve of the nanowire has a significantly higher slope, both positive and negative, with respect to the one of the SCF with "real" bridges. In particular, the maximum dispersion value reached is about $1425 \mathrm{ps} /(\mathrm{km} \cdot \mathrm{nm})$ around $2450 \mathrm{~nm}$ and about $136 \mathrm{ps} /(\mathrm{km} \cdot \mathrm{nm})$ around $2075 \mathrm{~nm}$ for the nanowire and the "real" SCF, respectively. The difference in the dispersion properties becomes lower and lower as the core radius becomes larger, since the chalcogenide glass core is large enough to completely contain the fundamental mode field, which is tightly confined in the high refractive index region at the center of the fiber cross-section, independently form the bridge presence. Notice that, as $r_{c}$ increases, the dispersion curves have almost the same slope and are overlapping at the shortest wavelengths in the considered range. For the largest core radius, that is $r_{c}=1.5 \mu \mathrm{m}$, the difference between the maximum $D$ parameter values is only about $110 \mathrm{ps} /(\mathrm{km} \cdot \mathrm{nm})$ at $3500 \mathrm{~nm}$, being about $220 \mathrm{ps} /(\mathrm{km} \cdot \mathrm{nm})$ for the chalcogenide glass nanowire. The fundamental mode field confinement in the $\mathrm{As}_{2} \mathrm{~S}_{3}$ core can explain also the behaviour of the dispersion curve of the SCFs with "real" bridges as a function of the core radius $r_{c}$. In particular, the influence of the core dimension on the fiber dispersion properties becomes negligible 

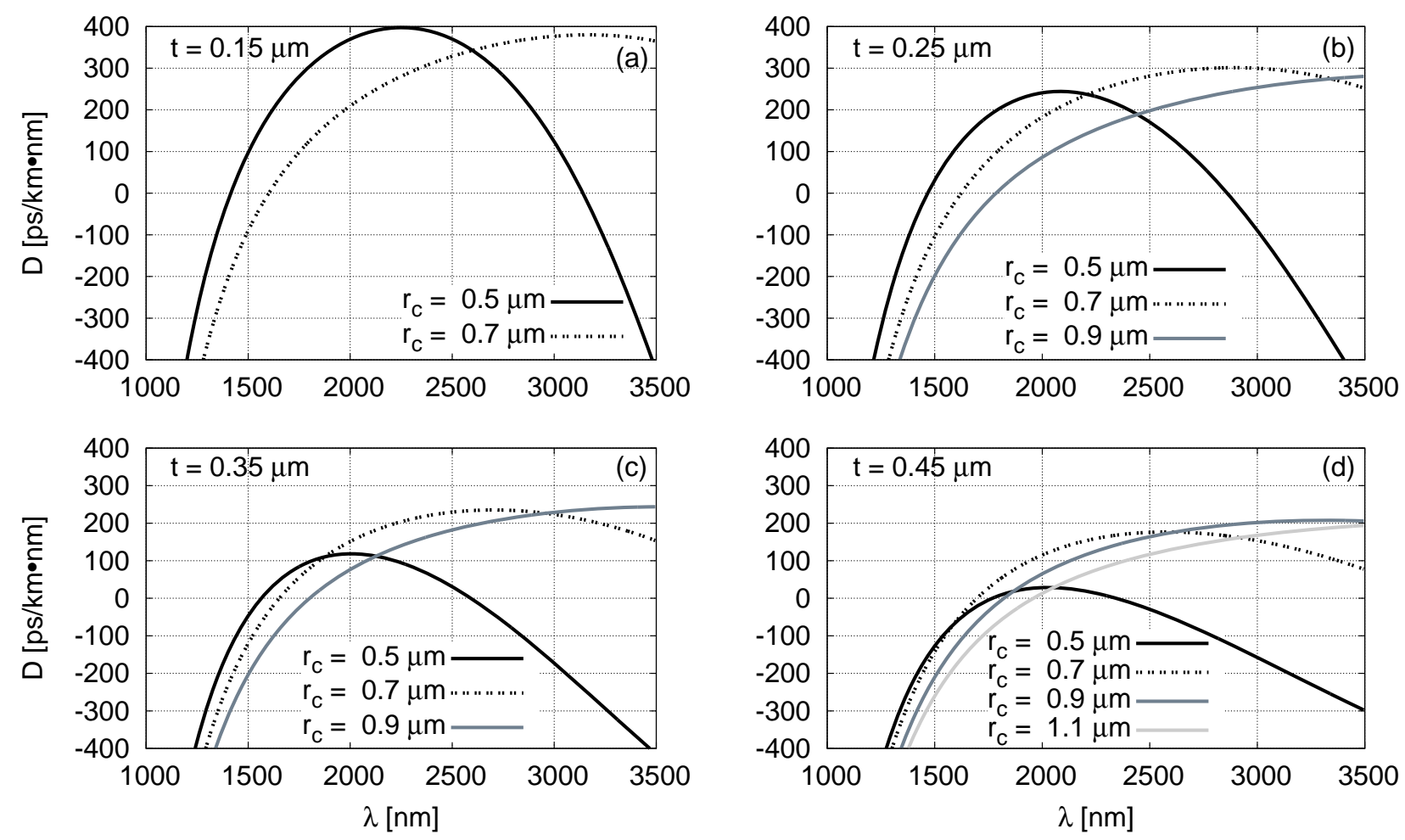

Figure 4. Dispersion parameter versus wavelength of the 5 -bridge SCFs with strut thickness $t$ of (a) $0.15 \mu \mathrm{m}$, (b) $0.25 \mu \mathrm{m}$, (c) $0.35 \mu \mathrm{m}$, and (d) $0.45 \mu \mathrm{m}$, and different values of the core radius $r_{c}$.

as the chalcogenide glass core enlarges, and for $r_{c} \geq 0.9 \mu \mathrm{m}$ all the SCF dispersion curves are almost overlapping in all the considered wavelength range. It is important to underline that when the core radius is large enough to provide a tight field confinement, with negligible leakage in the struts, the dispersion properties of the fibers with "ideal" bridges are very similar to the ones of the nanowire, since, for a certain $r_{c}$ value, the two guiding structures have exactly the same core area. On the contrary, a slightly larger core region is provided by the parabolic-shaped interconnecting regions in the fibers with "real" struts, thus causing lower $D$ parameter values, especially at longer wavelengths, for $r_{c}>1.1 \mu \mathrm{m}$. For lower $r_{c}$ values, since there are significant fundamental mode field tails in the struts, the dispersion curves of the structures with bridges, regardless of their shape, are more similar, and a stronger difference exist with respect to the nanowire properties.

After this preliminary study, the $\mathrm{As}_{2} \mathrm{~S}_{3} \mathrm{SCF}$ with 3 "real"-shaped bridges has been considered as the starting point for a throughout analysis of the influence of the main cross-section characteristics on the fiber dispersion properties. Simulation results obtained by changing the strut thickness $t$ for different $r_{c}$ values are reported in Fig. 3, 4 and 5 for SCFs with 3, 5 and 6 bridges, respectively. It is important to underline that, bearing in mind the technological feasibility of the designed fibers, thinner bridges have been considered only for fibers with smaller core. For example, struts with $t=0.15 \mu \mathrm{m}$ have been chosen only when $r_{c}$ is equal to $0.5 \mu \mathrm{m}$ or $0.7 \mu \mathrm{m}$, regardless of the bridge number.

By comparing the dispersion curves shown in Fig. 3(a)-(d), it is possible to notice that all the 3-bridge SCFs with the smallest core, that is $r_{c}=0.5 \mu \mathrm{m}$, have two ZDWs in the considered wavelength range. In particular, the first one, called $\lambda_{Z D W, 1}$, is around $1500 \mathrm{~nm}$, independently to the strut width. It is important to underline that in these small-core fibers the thickness $t$ of the glass bridges has a strong impact on the behavior of the dispersion parameter as a function of the wavelength. In particular, wider struts cause an overall decrease of $D$ parameter values. For example, when $r_{c}=0.5 \mu \mathrm{m}$, the maximum $D$ value is over $300 \mathrm{ps} /(\mathrm{km} \cdot \mathrm{nm})$ for the SCF with $t=0.15 \mu \mathrm{m}$, and only $130 \mathrm{ps} /(\mathrm{km} \cdot \mathrm{nm})$ for the one with $t=0.45 \mu \mathrm{m}$. More interestingly, in the fibers with $r_{c}=0.5 \mu \mathrm{m}$ the increase of the bridge thickness causes the distance between the two ZDWs to narrow, mostly by blue-shifting the second ZDW, called $\lambda_{Z D W, 2}$. As a result, $\lambda_{Z D W, 2}=2763 \mathrm{~nm}$ when $t=0.45 \mu \mathrm{m}$, while it is 

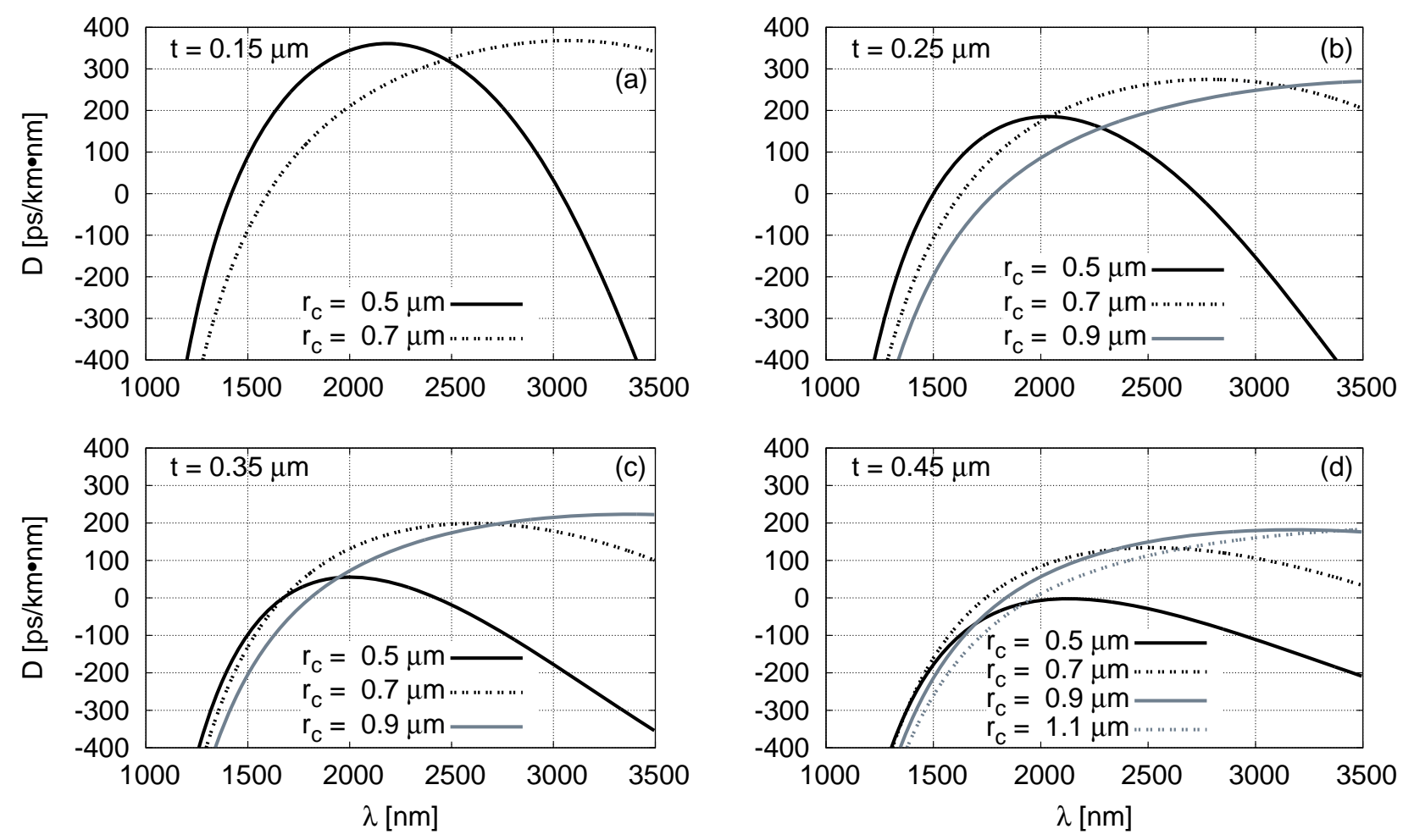

Figure 5. Dispersion parameter versus wavelength of the 6-bridge SCFs with strut thickness $t$ of (a) $0.15 \mu \mathrm{m}$, (b) $0.25 \mu \mathrm{m}$, (c) $0.35 \mu \mathrm{m}$, and (d) $0.45 \mu \mathrm{m}$, and different values of the core radius $r_{c}$.
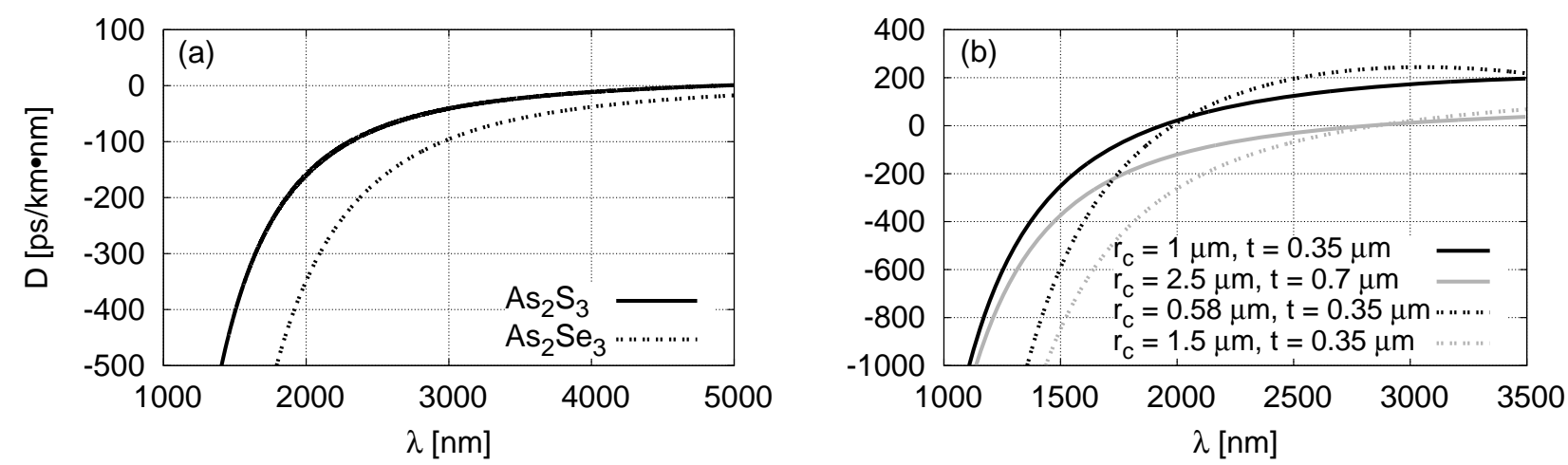

Figure 6. (a) Dispersion parameter versus wavelength of the two chalcogenide glasses $\mathrm{As}_{2} \mathrm{~S}_{3}$ and $\mathrm{As}_{2} \mathrm{Se}_{3}$. (b) Dispersion curves of the 3-bridge SCFs, made of $\mathrm{As}_{2} \mathrm{~S}_{3}$ (solid line) and $\mathrm{As}_{2} \mathrm{Se}_{3}$ (dashed line), designed to have the ZDW around $2000 \mathrm{~nm}$ and $2800 \mathrm{~nm}$.

$3489 \mathrm{~nm}$ for $t=0.15 \mu \mathrm{m}$. Simulation results reported in Fig. 3 demonstrate also that, as the chalcogenide glass core enlarges, the dispersion curves of the 3-bridge SCFs red-shift and the anomalous dispersion regime becomes wider. Moreover, the dispersion curve is progressively flattened and $\lambda_{Z D W, 2}$ is shifted outside the considered wavelength range. The influence of the bridge thickness is greatly reduced in the SCFs with larger core, and no significant effects are observed when $r_{c} \geq 0.9 \mu \mathrm{m}$.

A further degree of freedom for the SCF dispersion engineering is provided by the choice of the number of bridges, which influences the shape of the core and, consequently, the confinement of the fundamental mode field. Looking at the results reported in Fig. 4(a)-(d) and in Fig. 5(a)-(d) for fibers with 5 and 6 bridges, respectively, it is possible to notice that the most significant effect due to the increased number of struts is that their thickness has a stronger impact on the dispersion properties, especially if small cores are considered. For example, by 
Table 1. Main characteristics of the 3-bridge SCFs, made of $\mathrm{As}_{2} \mathrm{~S}_{3}$ and $\mathrm{As}_{2} \mathrm{Se}_{3}$, designed for pumping at $2000 \mathrm{~nm}$ and $2800 \mathrm{~nm}$ to obtain mid-IR SC generation.

\begin{tabular}{|c|c|c|c|c|}
\hline \multicolumn{5}{|c|}{$\mathrm{As}_{2} \mathrm{~S}_{3}$} \\
\hline \hline$r_{c}[\mu \mathrm{m}]$ & $t[\mu \mathrm{m}]$ & ZDW $[\mathrm{nm}]$ & $\lambda_{\text {pump }}[\mathrm{nm}]$ & $\gamma_{N L}$ at $\lambda_{\text {pump }}[1 /(\mathrm{W} \cdot \mathrm{km})]$ \\
\hline 1 & 0.35 & 1935 & 2000 & 4624 \\
\hline 2.5 & 0.7 & 2825 & 2850 & 595 \\
\hline \hline \multicolumn{5}{|c|}{$\mathrm{As}_{2} \mathrm{Se}_{3}$} \\
\hline \hline$r_{c}[\mu \mathrm{m}]$ & $t[\mu \mathrm{m}]$ & ZDW $[\mathrm{nm}]$ & $\lambda_{\text {pump }}[\mathrm{nm}]$ & $\gamma_{N L}$ at $\lambda_{\text {pump }}[1 /(\mathrm{W} \cdot \mathrm{km})]$ \\
\hline 0.58 & 0.35 & 1982 & 2000 & 36460 \\
\hline 1.5 & 0.35 & 2855 & 2800 & 5625 \\
\hline
\end{tabular}

comparing the SCFs with 3 and 5 bridges and $r_{c}=0.5 \mu \mathrm{m}$, it is clear that higher $D$ parameter values, up to $400 \mathrm{ps} /(\mathrm{km} \cdot \mathrm{nm})$, can be achieved with $t=0.15 \mu \mathrm{m}$ in the fiber with more struts. The situation is reversed for the smallest-core designs with large struts, being, for example, the maximum dispersion parameter value about $130 \mathrm{ps} /(\mathrm{km} \cdot \mathrm{nm})$ in the 3-bridge SCF with $t=0.45 \mu \mathrm{m}$ and less than $50 \mathrm{ps} /(\mathrm{km} \cdot \mathrm{nm})$ for the 5 -bridge fiber with the same geometric parameters. Furthermore, in the smallest-core SCFs with 5 struts the change of $t$ has a stronger effect on $\lambda_{Z D W, 1}$, which is significantly red-shifted by the increase of the bridge width, while the first ZDW is almost constant in the SCFs with 3 bridges. A larger dispersion slope is also generally observed, yielding to a narrowing of the distance between the two ZDWs, down to about $570 \mathrm{~nm}$ for the SCF with $r_{c}=0.5 \mu \mathrm{m}$ and $t=0.45 \mu \mathrm{m}$. The effect of the bridge thickness gradually fades out with the increase of the core size, and becomes negligible for $r_{c}>0.9 \mu \mathrm{m}$, as already observed for SCFs with fewer struts. However, it is important to underline that also for these large core radius, the number of bridges in the cladding is almost unimportant. Finally, for what concerns 6-bridge SCF dispersion properties, results show that, for small core radius, that is for fibers whose dispersion curve is still influenced by the strut thickness, the dispersion parameter values are slightly lower than in the 5-bridge counterparts, for any given value of $t$. It is important to notice that, by choosing $r_{c}=0.5 \mu \mathrm{m}$ and $t=0.45 \mu \mathrm{m}$, the SCF with 6 bridges operates in normal dispersion regime in all the considered wavelength range. Again, the core widening causes the effect of $t$ on the dispersion properties to become less significant, as the curves converge on those already found for SCFs with 3 and 5 bridges.

\section{SCF DESIGN FOR MID-IR SC GENERATION}

Simulation results of the thorough analysis on dispersion properties of $\mathrm{As}_{2} \mathrm{~S}_{3}$ SCFs presented in the previous Section provide important dispersion engineering guidelines which have been exploited to design nonlinear chalcogenide fibers suitable for SC generation in the mid-IR. In particular, the core radius and the bridge thickness of SCFs with 3 bridges, which are the easiest to fabricate, have been properly changed to obtain a ZDW around the wavelengths of $2000 \mathrm{~nm}$ and $2800 \mathrm{~nm}$, which can be both considered for efficient pumping for the SC generation. Besides $\mathrm{As}_{2} \mathrm{~S}_{3}$, another chalcogenide glass, that is $\mathrm{As}_{2} \mathrm{Se}_{3}$, has been taken into account, in order to increase the degrees of freedom for the dispersion engineering. As shown in Fig. 6(a), which reports the two glass dispersion curves in the wavelength range between $1000 \mathrm{~nm}$ and $5000 \mathrm{~nm}$, the latter chalcogenide material provides a ZDW which is longer than the $\mathrm{As}_{2} \mathrm{~S}_{3}$ one, which is around $4900 \mathrm{~nm}$. As a consequence, it is necessary to significantly blue-shift the $\mathrm{As}_{2} \mathrm{Se}_{3} \mathrm{ZDW}$ towards the desired wavelength range by introducing a strong waveguide dispersion component through the SCF design, which is obtained, according to previous results, with a reduction of the core radius.

Fig. 6(b) reports the dispersion curves of the SCFs, made of both $\mathrm{As}_{2} \mathrm{~S}_{3}$ and $\mathrm{As}_{2} \mathrm{Se}_{3}$, which have been designed to have a proper ZDW for SC generation pumping around $2000 \mathrm{~nm}$ and $2800 \mathrm{~nm}$. The main characteristics of the proposed fibers are summarized in Tab. 1. It is important to underline that, as expected, both the $\mathrm{As}_{2} \mathrm{Se}_{3}$ fibers have a core which is smaller than the one of the SCFs made of $\mathrm{As}_{2} \mathrm{~S}_{3}$. Moreover, larger core fibers provide a longer ZDW, regardless of the chalcogenide glass chosen. Finally, notice that all the designed SCFs offer a 
high nonlinear coefficient $\gamma_{N L}$, that is between $5951 /(\mathrm{W} \cdot \mathrm{km})$ and $364601 /(\mathrm{W} \cdot \mathrm{km})$, so the enhanced nonlinear properties of the proposed chalcogenide fibers can be successfully exploited for the SC generation.

\section{CONCLUSION}

The dispersion properties of chalcogenide suspended-core fibers have been thoroughly analyzed by means of a full-vector modal solver based on the finite-element method. After showing the importance of taking into account the glass struts for the numerical modeling of the guiding characteristics of these microstructured fibers, the possibility to widely engineer their dispersion curve by acting on the core radius, and on the number and thickness of the glass bridges has been investigated. A wide range of geometric parameter values have been considered, in order to prove guidelines for the design of highly nonlinear suspended-core fibers to operate in several applications relying on different pumping sources. Finally, the fiber design has been optimized in order to obtain proper dispersion properties around $2000 \mathrm{~nm}$ and $2800 \mathrm{~nm}$ for efficient pumping for mid-IR supercontinuum generation.

\section{ACKNOWLEDGMENTS}

The authors wish to thank Romain Dauliat, Laurent Brilland and Johann Troles for providing useful information about chalcogenide glasses and chalcogenide fiber fabrication.

\section{REFERENCES}

[1] Coscelli, E., Sozzi, M., Poli, F., Passaro, D., Cucinotta, A., Selleri, S., Corradini, R., and Marchelli, R., "Toward A Highly Specific DNA Biosensor: PNA-Modified Suspended-Core Photonic Crystal Fibers," Selected Topics in Quantum Electronics, IEEE Journal of 16, 967-972 (July 2010).

[2] "Sensing with suspended-core optical fibers," Optical Fiber Technology 16(6), 343 - 356 (2010).

[3] Chu, F., Tsiminis, G., Spooner, N. A., and Monro, T. M., "Explosives detection by fluorescence quenching of conjugated polymers in suspended core optical fibers," Sensors and Actuators B: Chemical 199, 22 - 26 (2014).

[4] Frazao, O., Aref, S., Baptista, J., Santos, J., Latifi, H., Farahi, F., Kobelke, J., and Schuster, K., "Fabry perot cavity based on a suspended-core fiber for strain and temperature measurement," Photonics Technology Letters, IEEE 21, 1229-1231 (Sept 2009).

[5] Kuan, P.-W., Li, K., Zhang, G., Wang, X., Zhang, L., Bai, G., Tsang, Y., and Hu, L., "Compact broadband amplified spontaneous emission in $\mathrm{Tm}^{3+}$-doped tungsten tellurite glass double-cladding single-mode fiber," Opt. Mater. Express 3, 723-728 (Jun 2013).

[6] Cheng, T., Usaki, R., Duan, Z., Gao, W., Deng, D., Liao, M., Kanou, Y., Matsumoto, M., Misumi, T., Suzuki, T., and Ohishi, Y., "Soliton self-frequency shift and third-harmonic generation in a four-hole $\mathrm{As}_{2} \mathrm{~S}_{5}$ microstructured optical fiber," Opt. Express 22, 3740-3746 (Feb 2014).

[7] Duhant, M., Renard, W., Canat, G., Nguyen, T. N., Smektala, F., Troles, J., Coulombier, Q., Toupin, P., Brilland, L., Bourdon, P., and Renversez, G., "Fourth-order cascaded Raman shift in AsSe chalcogenide suspended-core fiber pumped at $2 \mu \mathrm{m}$," Opt. Lett. 36, 2859-2861 (Aug 2011).

[8] Savelii, I., Mouawad, O., Fatome, J., Kibler, B., Désévédavy, F., Gadret, G., Jules, J.-C., Bony, P.-Y., Kawashima, H., Gao, W., Kohoutek, T., Suzuki, T., Ohishi, Y., and Smektala, F., "Mid-infrared 2000$\mathrm{nm}$ bandwidth supercontinuum generation in suspended-core microstructured Sulfide and Tellurite optical fibers," Opt. Express 20, 27083-27093 (Nov 2012).

[9] Gao, W., El-Amraoui, M., Liao, M., Kawashima, H., Duan, Z., Deng, D., Cheng, T., Suzuki, T., Messaddeq, Y., and Ohishi, Y., "Mid-infrared supercontinuum generation in a suspended-core $\mathrm{As}_{2} \mathrm{~S}_{3}$ chalcogenide microstructured optical fiber," Opt. Express 21, 9573-9583 (Apr 2013).

[10] Harbold, J. M., Ilday, F. Ö., Wise, F. W., Sanghera, J. S., Nguyen, V. Q., Shaw, L. B., and Aggarwal, I. D., "Highly nonlinear As-S-Se glasses for all-optical switching," Opt. Lett. 27, 119-121 (Jan 2002).

[11] Désévédavy, F., Renversez, G., Brilland, L., Houizot, P., Troles, J., Coulombier, Q., Smektala, F., Traynor, N., and Adam, J.-L., "Small-core chalcogenide microstructured fibers for the infrared," Appl. Opt. 47, 6014-6021 (Nov 2008). 
[12] Ta'eed, V., Baker, N. J., Fu, L., Finsterbusch, K., Lamont, M. R. E., Moss, D. J., Nguyen, H. C., Eggleton, B. J., Choi, D.-Y., Madden, S., and Luther-Davies, B., "Ultrafast all-optical chalcogenide glass photonic circuits," Opt. Express 15, 9205-9221 (Jul 2007).

[13] Rudy, C. W., Marandi, A., Vodopyanov, K. L., and Byer, R. L., "Octave-spanning supercontinuum generation in in situ tapered $\mathrm{As}_{2} \mathrm{~S}_{3}$ fiber pumped by a thulium-doped fiber laser," Opt. Lett. 38, 2865-2868 (Aug 2013).

[14] Yue, Y., Zhang, L., Yan, Y., Ahmed, N., Yang, J.-Y., Huang, H., Ren, Y., Dolinar, S., Tur, M., and Willner, A. E., "Octave-spanning supercontinuum generation of vortices in an $\mathrm{As}_{2} \mathrm{~S}_{3}$ ring photonic crystal fiber," Opt. Lett. 37, 1889-1891 (Jun 2012).

[15] Wei, C., Zhu, X., Norwood, R. A., Song, F., and Peyghambarian, N., "Numerical investigation on high power mid-infrared supercontinuum fiber lasers pumped at $3 \mu \mathrm{m}$," Opt. Express 21, 29488-29504 (Dec 2013).

[16] Zakery, A. and Elliott, S. R., [Optical Nonlinearities in Chalcogenide Glasses and their Applications], Springer Series in Material Science, Dordrecht (2007).

[17] Slusher, R. E., Lenz, G., Hodelin, J., Sanghera, J., Shaw, L. B., and Aggarwal, I. D., "Large Raman gain and nonlinear phase shifts in high-purity $\mathrm{As}_{2} \mathrm{Se}_{3}$ chalcogenide fibers," J. Opt. Soc. Am. B 21, 1146-1155 (Jun 2004).

[18] Poli, F., Cucinotta, A., and Selleri, S., [Photonic Crystal Fibers. Properties and Applications], Springer Series in Material Science, Dordrecht (2007).

[19] El-Amraoui, M., Fatome, J., Jules, J. C., Kibler, B., Gadret, G., Fortier, C., Smektala, F., Skripatchev, I., Polacchini, C., Messaddeq, Y., Troles, J., Brilland, L., Szpulak, M., and Renversez, G., "Strong infrared spectral broadening in low-loss As-S chalcogenide suspended core microstructured optical fibers," Opt. Express 18, 4547-4556 (Mar 2010).

[20] Gao, W., Liao, M., Yan, X., Kito, C., Kohoutek, T., Suzuki, T., El-Amraoui, M., Jules, J.-C., Gadret, G., Désévédavy, F., Smektala, F., and Ohishi, Y., "Visible Light Generation and Its Influence on Supercontinuum in Chalcogenide $\mathrm{As}_{2} \mathrm{~S}_{3}$ Microstructured Optical Fiber," Applied Physics Express 4(10), 102601 (2011).

[21] Rodney, W., Malitson, I., and King, T., "Refractive index of Arsenic Trisulfide," J. Opt. Soc. Am. 48, 633-636 (Sep 1958).

[22] http://www. amorphousmaterials.com/.

[23] Vincetti, L., Maini, M., Poli, F., Cucinotta, A., and Selleri, S., "Numerical analysis of hollow core photonic band gap fibers with modified honeycomb lattice," Optical and Quantum Electronics 38(9-11), 903-912 (2006).

[24] El-Amraoui, M., Gadret, G., Jules, J. C., Fatome, J., Fortier, C., Désévédavy, F., Skripatchev, I., Messaddeq, Y., Troles, J., Brilland, L., Gao, W., Suzuki, T., Ohishi, Y., and Smektala, F., "Microstructured chalcogenide optical fibers from $\mathrm{As}_{2} \mathrm{~S}_{3}$ glass: towards new IR broadband sources," Opt. Express 18, 2665526665 (Dec 2010). 